\title{
Le développement des télécommunications dans les Suds. Retour sur une décennie de diffusion des TIC en Afrique de l'Ouest et au Maghreb
}

Ahmed Dahmani et Jean-Michel Ledjou

\section{(2) OpenEdition \\ Journals}

Édition électronique

URL : http://journals.openedition.org/ticetsociete/1057

DOI : 10.4000/ticetsociete.1057

\section{Éditeur}

Association ARTIC

\section{Référence électronique}

Ahmed Dahmani et Jean-Michel Ledjou, « Le développement des télécommunications dans les Suds. Retour sur une décennie de diffusion des TIC en Afrique de l'Ouest et au Maghreb », tic\&société [En ligne], Vol. 5, n²-3 | 2e sem. 2011 / 1er sem. 2012, mis en ligne le 20 juin 2012, consulté le 01 mai 2019. URL : http://journals.openedition.org/ticetsociete/1057 ; DOI : 10.4000/ticetsociete.1057 
tic\&société - 5 (2-3), $2^{\text {ème }}$ semestre 2011 - $1^{\text {er }}$ semestre 2012

\title{
Le développement des télécommunications dans les Suds Retour sur une décennie de diffusion des TIC en Afrique de l'Ouest et au Maghreb
}

\author{
Ahmed DAHMANI \\ AMETIS - Plateau du Moulon - 91400 Orsay. \\ Téléphone professionnel : +33 (0) 1-69-33-61-24 - Université Paris- Sud /XI \\ ahmed.dahmani@psud.fr \\ Jean-Michel LEDJOU \\ AMETIS - Plateau du Moulon - 91400 Orsay. \\ Téléphone professionnel : +33 (0) 1-69-33-61-23 - Université Paris- Sud /XI \\ jean-michel.ledjou@u-psud.fr
}

Ahmed Dahmani est économiste. II exerce en tant que maître de conférences à I'IUT d'Orsay, Université Paris-Sud. II dirige le laboratoire de recherches AMETIS au sein du Collège d'Etudes Interdisciplinaires (CEI/UPS). II enseigne en tant que Professeur associé dans le cadre d'un Master sur le partenariat EUROMED à I'Institut Europe-Maghreb de I'Université Paris 8 à Saint Denis. II a été enseignant à l'Université de Tizi-Ouzou(Algérie) de 1979 à 1994.

Jean-Michel Ledjou est maître de conférences à l'Université Paris-Sud (IUT d'Orsay), spécialiste des Sciences de l'information et de la communication. Membre du CEI / AMETIS ses recherches actuelles portent sur les rapports entre communication et développement dans les pays du Sud. II mène également des travaux sur le lien entre les médias et la démocratie. 


\title{
Le développement des télécommunications dans les Suds Retour sur une décennie de diffusion des TIC en Afrique de l'Ouest et au Maghreb
}

\begin{abstract}
Résumé
Dans les Suds, et notamment en Afrique lors de la dernière décennie, les TIC connaissent une croissance considérable. Cet essor présente cependant de fortes disparités entre les différents pays. L'article a pour objectif d'analyser les politiques et les logiques de diffusion à l'œuvre en Afrique de l'Ouest et au Maghreb au cours de ces dix dernières années.
\end{abstract}

Mots-clés : Afrique, diffusion, investisseurs, marché, politiques publiques, TIC.

\begin{abstract}
During the last decade, in the southern developing countries, particularly in Africa, ICTs'growth is significant, as well as the inequalities between countries. This article aims at analyzing, and comparing the ICTs'dissemination policies implemented in West and North Africa during the past ten years.
\end{abstract}

Keywords : Africa, dissemination, investissor, market, public policies, ICT's

\begin{abstract}
Resumen
En el Sur, particularmente en África en el momento de la última década, las TIC conocen un crecimiento considerable. Este auge ofrece sin embargo enormes disparidades entre los diferentes países. El artículo tiene como objetivo analizar las políticas y las lógicas de difusión en obra en África occidental y en el Magreb en el curso de estos diez últimos años.
\end{abstract}

Palabras clave: África, difusiones, inversores, mercado, políticas públicas, TIC. 
Le développement des télécommunications dans les Suds

Retour sur une décennie de diffusion des TIC en Afrique de l'Ouest et au Maghreb

\section{Introduction}

Dans les Suds, et notamment en Afrique de l'Ouest et au Maghreb, les technologies de l'information et de la communication (TIC) ont connu une forte croissance lors de la dernière décennie. Les TIC ont également permis de mettre au jour des dynamiques à l'opposé de l'image figée que d'aucuns peuvent avoir du continent : des événements politiques en Tunisie et en Égypte à la place qu'elles tiennent dans les économies africaines, de nombreux exemples montrent que les technologies de l'information se sont imposées dans l'accompagnement des mutations à l'œuvre en Afrique. Mais qu'en est-il des politiques de diffusion?

Notre propos, qui se focalisera principalement sur l'Afrique de l'Ouest et le Maghreb, vise à analyser d'un point de vue socio-économique les politiques et les logiques de diffusion établies au cours des dix dernières années dans ces régions. Nous porterons un regard croisé sur ce phénomène, celui d'un économiste et celui d'un spécialiste des sciences de l'information et de la communication. L'étude, qui veut mettre en évidence l'indifférenciation des politiques poursuivies sur le continent, s'appuie sur l'analyse de travaux de recherche, de rapports produits par I'UIT et I'OCDE ainsi que sur nos propres observations.

\section{Les effets de la réforme des télécommunications sur les marchés africains}

L'ouverture aux TIC dans les pays du Sud, comme dans ceux du Nord, s'est déroulée dans le cadre de la libéralisation du secteur des télécommunications : privatisation des opérateurs historiques et déréglementation globale du marché. À de rares exceptions près en Afrique, cette réforme, présentée par les chantres du millénarisme technologique comme indispensable au développement même des technologies de l'information, a le plus souvent été conduite à marche forcée sous la houlette des institutions et des bailleurs de fonds internationaux (UIT, BM, FMI, OMC, OCDE, etc.). Ceux-ci ont affirmé que, pour développer favorablement le marché des TIC, la responsabilité en matière d'investissement devait revenir au secteur privé, et la régulation des marchés à une instance de régulation sinon autonome du moins indépendante vis-à-vis de l'État [BAD/OCDE, 2009]. D'une façon générale, la libéralisation du secteur des télécommunications s'est concrétisée par une série de réformes mettant en 
Ahmed DAHMANI et Jean-Michel LEDJOU

évidence une standardisation des prescriptions qui, dans la lignée des programmes d'ajustement structurel, ont pu être remises en cause par la société civile, notamment parce qu'elles fragilisent les opérateurs historiques et font la part belle aux investisseurs étrangers. [Sagna, 2007].

\subsection{Des marchés attractifs pour les investisseurs étrangers}

Les opérateurs internationaux voient le continent africain comme un territoire à conquérir à un moment où les marchés du Nord arrivent à saturation. Avec un taux de pénétration du mobile autour de $40 \%$ en $2010^{1}$, le potentiel de croissance de l'Afrique reste fort d'autant que la population augmente à un rythme deux fois plus élevé que dans les autres régions du monde ${ }^{2}$. La demande interne est par ailleurs soutenue et le secteur offre des opportunités de développement économique en lien avec le Nord.

Le premier effet de la libéralisation du secteur des télécommunications a trait à la configuration du marché : le nombre de réseaux mobiles en Afrique a triplé en sept ans. En 2002-2003, seuls douze pays africains n'avaient qu'un seul opérateur de téléphonie mobile, tandis que la concurrence s'établissait entre deux opérateurs dans vingt et un pays, entre trois dans huit pays, entre quatre dans six pays et entre cinq opérateurs dans deux pays. En 2007, selon les chiffres globaux fournis par I'UIT, le niveau de concurrence s'est sensiblement accru et les réseaux mobiles ne font l'objet de monopole qu'à hauteur de $9 \%$. Le marché de l'Internet est lui aussi relativement ouvert avec l'établissement de nombreux fournisseurs d'accès privés aux côtés des opérateurs historiques qui détiennent encore $11 \%$ du marché africain. Seul le marché de la téléphonie fixe demeure encore monopolisé, dans de nombreux pays, par les opérateurs historiques qui en contrôlent $45 \%$. La raison en est simple : si les opérateurs étrangers ont investi de façon quasi exclusive dans le secteur mobile, c'est parce qu'il leur permet d'engranger de substantiels bénéfices ${ }^{3}$ sans grever leur budget. "Le niveau des dépenses d'investissement et de fonctionnement en Afrique est inférieur à celui d'Amérique Latine, des Caraïbes et d'Asie. Parallèlement, les opérateurs africains de téléphonie mobile obtiennent de bons résultats en termes de trésorerie, avec des recettes supérieures aux autres régions. Les retours sur investissement sont attrayants pour ceux que les

\footnotetext{
1 /www.itu.int/fr/

${ }^{2}$ Selon les démographes de la Banque mondiale (http://web.worldbank.org )

3 En 2008, les abonnements à la téléphonie mobile et les recettes d'Orange en Afrique ont progressé respectivement de $42,5 \%$ et $17 \%$, contre 28 et $8,3 \%$ pour la totalité du groupe dans le monde, in : BAD/OCDE, op.cit., p.109.
} 
Le développement des télécommunications dans les Suds

Retour sur une décennie de diffusion des TIC en Afrique de l'Ouest et au Maghreb

réseaux africains intéressent, même si la concurrence s'intensifie progressivement » [BAD/OCDE, 2009].

Tableau 1 : Investisseurs stratégiques dans le secteur de la téléphonie mobile, 2007

\begin{tabular}{|l|l|l|l|}
\hline & \multicolumn{1}{|c|}{$\begin{array}{c}\text { Abonnés } \\
\text { (milliers) }\end{array}$} & \multicolumn{1}{|c|}{$\begin{array}{c}\text { Nombre de } \\
\text { pays }\end{array}$} & \multicolumn{1}{c|}{$\begin{array}{c}\text { Recettes } \\
\text { (milliers \$ US) }\end{array}$} \\
\hline $\begin{array}{l}\text { MTN (Afrique du } \\
\text { Sud) }\end{array}$ & 49837 & 16 & 9040 \\
\hline $\begin{array}{l}\text { Vodacom (Afrique } \\
\text { du Sud) }\end{array}$ & 33041 & 5 & 5818 \\
\hline Orascom (Égypte) & 32394 & 4 & 3761 \\
\hline $\begin{array}{l}\text { Zain ; Celtel } \\
\text { (Koweit) }\end{array}$ & 30171 & 15 & 3957 \\
\hline $\begin{array}{l}\text { Vodafone } \\
\text { (Royaume-Uni) }\end{array}$ & 22578 & 2 & 2075 \\
\hline $\begin{array}{l}\text { Vivendi/Maroc } \\
\text { Télécom }\end{array}$ & 11948 & 4 & 2336 \\
\hline $\begin{array}{l}\text { Luxembourg } \\
\text { Télécom }\end{array}$ & 5632 & 12 & 1795 \\
\hline $\begin{array}{l}\text { Milicom } \\
\text { (Luxembourg) }\end{array}$ & 1500 & 7 & 477 \\
\hline $\begin{array}{l}\text { MOÓ } \\
\text { (Émirats Arabes } \\
\text { Unis) }\end{array}$ & 211893 & 7 & -- \\
\hline Total & 542 & & \\
\hline
\end{tabular}

Source : UIT, 2008. 


\subsection{Une demande soutenue}

En dépit d'une croissance disparate et inégale entre les différents segments des TIC et les différents pays, l'engouement des Africains pour ces technologies est réel. Ces dernières ont connu une croissance forte depuis l'an 2000.

Tableau 2. Les TIC en Afrique entre 2000 et 2007

\begin{tabular}{|c|c|c|c|c|c|c|c|c|}
\hline & & Population & $\begin{array}{l}\text { Internet } \\
\text { (Utilisate }\end{array}$ & & $\begin{array}{l}\text { Téléphoni } \\
\text { (lignes pri }\end{array}$ & $\begin{array}{l}\text { ixe } \\
\text { pales) }\end{array}$ & Téléphoni & obile \\
\hline Zone & & Millions & Milliers & $\%$ & Milliers & $\%$ & Milliers & $\%$ \\
\hline $\mathrm{AFN}^{*}$ & 2000 & 137,89 & 710,0 & 0,52 & 10125,3 & 7,35 & 3883,2 & 2,82 \\
\hline & 2007 & 157,07 & 21402,2 & 13,64 & 18671,0 & 11,91 & 83865,0 & 53,39 \\
\hline $\mathrm{AFS}^{* *}$ & 2000 & 43,69 & 2400,0 & 5,5 & 4961,7 & 11,36 & 8308,0 & 19,02 \\
\hline & 2007 & 48,58 & 5100,0 & 10,75 & 4642,0 & 9,56 & 42300,0 & 87,08 \\
\hline $\mathrm{ASS}^{* \star *}$ & 2000 & 609,57 & 1302,2 & 0,21 & 4567,6 & 0,75 & 3373,1 & 0,56 \\
\hline & 2007 & 758,04 & 23904,2 & 3,23 & 12098,3 & 1,65 & 138310 & 18,28 \\
\hline Total & 2000 & 791,15 & 4412,2 & 0,56 & 19654,7 & 2,5 & 15564,2 & 1,98 \\
\hline & 2007 & 963,68 & 50406,4 & 5,34 & 35411,3 & 3,77 & 264475,0 & 27,48 \\
\hline
\end{tabular}

Source : Tableau élaboré par les auteurs à partir des rapports sur les télécommunications en Afrique (2002 et 2008) de l'UIT

* Afrique du Nord (Algérie, Égypte, Libye, Maroc, Tunisie)

** Afrique du Sud

${ }^{* * *}$ Afrique subsaharienne : (y compris Afrique centrale et australe)

L'évolution des connexions à l'Internet est impressionnante. Entre 2000 et 2007 , elles ont progressé de $874,6 \%$, soit près de 4 fois la progression 
Le développement des télécommunications dans les Suds

Retour sur une décennie de diffusion des TIC en Afrique de l'Ouest et au Maghreb

mondiale $(244,7 \%)^{4}$. Cette croissance cache cependant mal la faible connectivité africaine dans le monde : 5,34\% (moyenne mondiale : 18,9) pour une population qui en représente $14,2 \%$, avec de fortes disparités entre les différentes régions. En Afrique du Nord, c'est au Maroc que la progression a été particulièrement spectaculaire : le nombre d'internautes est passé de 120000 en 2000 à 7,3 millions en 2007 (soit un multiple de 60). En 2006, le taux d'utilisateurs en Afrique subsaharienne oscille entre 0,28 \% au Niger, 0,50 \% au Mali, 0,54 \% au Burkina Faso, 1,63 \% en Côte d'Ivoire et 5,45 \% au Sénégal. La faiblesse de la connectivité est d'abord due à la disproportion des tarifs de connexion par rapport aux revenus et ce malgré des baisses importantes et des promotions régulières. Selon I'UIT, "un panier mensuel défini sur la base de $20 \mathrm{~h}$ d'utilisation d'Internet coûte plus de 50 \$ US en Afrique, chiffre presque deux fois supérieur au tarif pratiqué dans la région classée au second rang [Amériques] et équivalent à près de $70 \%$ du revenu moyen par habitant de l'Afrique sub-saharienne ${ }^{5}$. À cela s'ajoute un faible taux d'équipement des ménages en ordinateurs (2,24 PCs/100 hab.). Et même si les conditions de connexion se sont améliorées dans de nombreux pays, notamment avec l'introduction de l'ADSL (c'est le cas des pays d'Afrique du Nord), celles-ci demeurent conditionnées à un coût élevé et tributaires d'infrastructures de base encore peu développées. Ainsi, comme le montre le tableau 2, la télédensité fixe demeure faible par rapport à la télédensité mobile. La téléphonie fixe a peu progressé ces dernières années et a même régressé dans certains pays comme l'Afrique du Sud. Au seuil de l'année 2008, il y avait 35 millions de lignes fixes sur le continent. La télédensité a progressé de seulement 1 point entre 2002 et 2007, passant de 2,8 à 3,8 avec les disparités traditionnelles (AFN : 11,9; AFS : 9,6 ; ASS : 1,6). Les opérations de privatisation, là où elles ont été menées, n'ont pas dynamisé le secteur et les opérateurs historiques ont eu tendance à augmenter le tarif des abonnements et le prix des communications locales pour parer au recul du trafic qui a profité à la téléphonie mobile.

Les obstacles dans l'accès à l'Internet sont contournés par les utilisateurs qui se connectent plutôt via des centres d'accès collectif, généralement appelés « cybercentres». Ces structures, relativement abordables en terme de tarifs, peuvent rester ouvertes 24 heures sur 24 et 7 jours sur 7 . En Afrique du Nord, seule la Tunisie se distingue des autres pays par un faible nombre d'accès publics en comparaison avec ses voisins : 0,3 pour 10000 habitants contre 0,66

\footnotetext{
${ }^{4}$ Source : http://www.internetworldstats.com/stats $\left.1 . h t m\right]$.

${ }^{5}$ UIT, base de données de réglementation sur les télécommunications dans le monde, Indicateurs des télécommunications/TIC africaines, 2008.
} 
au Maroc et 1,4 en Algérie $^{6}$. Au Sénégal, on compte près de 800 points d'accès publics à l'Internet, soit 0,66 pour 10000 habitants $^{7}$.

Mais c'est bien la téléphonie mobile qui soulève l'enthousiasme populaire le plus important. Un engouement qui fait dépenser aux ménages africains $10 \%$ de leur revenu mensuel contre seulement $3 \%$ pour les ménages des pays développés [Tcheng et al., 2009]. En 2007, le nombre d'abonnés au mobile en Afrique représente plus de sept fois les abonnés du fixe (264.475 millions contre 35,4 millions) ${ }^{8}$. Mieux, la plupart des abonnés au mobile n'ont jamais disposé d'un téléphone fixe. Plusieurs raisons expliquent ce phénomène. Du côté des usagers, la diversité et la qualité des services offerts sont, à plus d'un titre, appréciées des clients. Ceux-ci n'ont plus à subir les longues listes d'attente comme ce fut le cas lorsqu'il s'agissait d'obtenir un raccordement à la téléphonie fixe. Ils peuvent aussi bénéficier du service de cession crédit-temps ou de minimessages de rappel gratuits. Enfin, la mise en place du système de prépaiement (près de $94 \%$ des utilisateurs selon I'UIT) se révèle bien adaptée au budget limité des ménages africains même si les tarifs pratiqués sont parmi les plus chers du monde. Le caractère fondamentalement aléatoire du « budget télécommunications " des ménages explique le succès d'un tel dispositif qui, soulignons-le au passage, institue une confortable avance de trésorerie pour les opérateurs : le service est payé avant même d'avoir été utilisé.

\subsection{Le secteur des services dynamisé}

Les TIC ont permis de développer l'offre de services. Dans le domaine de l'Internet, par exemple, de jeunes diplômés ou des passionnés d'informatique montent leur petite entreprise avec peu de moyens. La structure la plus emblématique de cette tendance est le cybercentre où plusieurs services sont offerts à la clientèle : navigation Internet, apprentissage informatique, création de sites, etc. Un peu partout dans les grandes agglomérations africaines essaiment des cybercentres pourvoyeurs de nouveaux emplois pour les populations locales ${ }^{9}$. Certains pays, comme le Maroc, la Tunisie ou le Sénégal, ont investi dans la mise en place de technopoles spécialisées dans les TIC afin de soutenir les activités de recherche et d'innovations et de favoriser l'attractivité des investissements étrangers dans le secteur des TIC. II serait prématuré d'en

\footnotetext{
${ }_{7}^{6}$ Calculs des auteurs selon les données fournies par les organismes officiels des trois pays.

7 Source : http://www.osiris.sn/article27.html

${ }^{8}$ UIT, Indicateurs des télécommunications/TIC africaines, 2008,

${ }^{9}$ Sur ce point, on pourra lire [Chéneau-Loquay., 2009], 
Le développement des télécommunications dans les Suds

Retour sur une décennie de diffusion des TIC en Afrique de l'Ouest et au Maghreb

tirer un quelconque bilan, mais on peut tout de même constater la dynamisation des activités d'infogérance: centres d'appels, secrétariat, édition, saisie de données, services bancaires et d'assurances, services informatiques, etc. Les entreprises du Nord voient dans ces pratiques une opportunité qui rend possible la délocalisation de services dans des pays où salaires et charges sont moins élevés. Ces activités créent de l'emploi pour les jeunes diplômés exclus du marché du travail local mais dans des créneaux peu qualifiés et en marge de l'évolution technologique. Mieux payés que dans d'autres secteurs d'activité, ces salariés font bien souvent figure de privilégiés. Mais le principal risque, comme semble l'indiquer l'expérience tunisienne, est que ces pôles deviennent de véritables enclaves économique et technologique, sans lien ni impact réels sur l'économie et la société [Mezouaghi, Perrat, 2007].

\section{Accélérer la diffusion : le global à l'épreuve du local}

Les stratégies de diffusion impulsées par les opérateurs internationaux se heurtent à la réalité locale qui traduit de grandes disparités dans la mise en place puis dans la gestion des politiques publiques.

\subsection{Les politiques publiques en question}

À partir des années quatre-vingt-dix, dans un contexte marqué par la rareté des ressources financières et par l'importation des TIC, les gouvernements ont vu une chance dans la privatisation des opérateurs historiques: la vente des licences d'exploitation a constitué un moyen d'encaisser des devises. Le processus de privatisation leur a également permis de s'affranchir des contraintes de la modernisation des infrastructures. Mais plusieurs États d'Afrique de l'Ouest ont éprouvé des difficultés à faire respecter le cahier des charges accepté par les opérateurs au moment de l'ouverture à la concurrence. Par ailleurs, plusieurs anomalies d'interconnexion entre réseaux ont été signalées, notamment à l'échelle de la téléphonie mobile. Cela s'est traduit soit par l'impossibilité d'établir une connexion entre deux réseaux concurrents, soit, plus fréquemment, par des coûts d'interconnexion particulièrement onéreux, contraignant les clients des opérateurs à souscrire deux abonnements distincts dans de nombreux pays. 
Est-ce aussi une autre conséquence des privatisations massives ? En tout cas, les opérateurs de télécommunication se sont prioritairement implantés dans les villes les plus peuplées, laissant ainsi à l'écart les zones rurales, nettement moins génératrices de profits.

Contrairement aux années de postindépendance, les stratégies de diffusion des TIC en Afrique ne sont pas l'apanage de l'État ni sa préoccupation principale, même si les discours publics sur le sujet ne cessent de scander l'importance des TIC dans le développement. Ainsi, la promesse technologique sustente-t-elle la rhétorique technophile des États et des organismes internationaux. Les discours institutionnels méritent pourtant d'être relativisés à la lumière des situations locales.

Les États éprouvent en effet parfois bien des difficultés à se détacher de la mainmise qu'ils exercent sur le secteur des télécommunications. Et les politiques publiques manquent souvent d'efficience. Cela se manifeste, par exemple, par de grandes disparités sur le plan des autorités de régulation. Celles-ci ne sont pas toutes totalement indépendantes, et quand bien même elles disposent d'une indépendance de fait, leur surface financière demeure limitée. C'est là un point délicat, car seul un État qui pratique la séparation des pouvoirs peut accepter de transférer ses compétences de régulation à un organe qui échappe à sa propre tutelle. Or, la pratique, relativement courante, de la collusion des intérêts supporte mal l'avènement sur le marché d'un tiers acteur dont la vocation est de sanctionner l'opacité liée aux procédures d'attribution et aux transactions opérées sur le marché. On comprend donc que la réforme des télécommunications ne se traduit pas systématiquement par un désengagement effectif de l'État mais par une modification/adaptation des formes de son intervention à travers les autorités de régulation. L'exemple du Maroc, érigé en modèle d'ouverture par I'UIT, est de ce point de vue édifiant. L'indépendance de l'ANRT est en effet toute relative : celle-ci est placée sous l'autorité du Premier ministre qui en nomme la plupart des membres et détermine son budget de fonctionnement. De plus, "il y a un risque pour les autorités de régulation nationale d'être trop faibles face aux opérateurs multinationaux " d'où la nécessité d'un regroupement "au niveau de grands ensembles régionaux de façon à renforcer leurs pouvoirs d'intervention" [Flichy, 2005].

Les États ne renvoient pas non plus toujours aux mêmes réalités. Certains pays, comme le Congo ou le Tchad, demeurent instables sur le plan politique et fragile sur le plan économique. D'autres, à l'instar de la Côte d'Ivoire, présentent une structure de marché extrêmement concurrentielle, notamment sur le plan de la téléphonie mobile. 
Le développement des télécommunications dans les Suds

Retour sur une décennie de diffusion des TIC en Afrique de l'Ouest et au Maghreb

Sur le marché de l'Internet, la fourniture d'accès est libre et ouverte au capital étranger dans bien des pays. C'est le cas du Maroc où les formalités pour devenir fournisseur d'accès à Internet (FAl) sont relativement simples mais où la structure de marché demeure particulièrement concentrée avec la constitution de structures quasi duopolistiques. Le marché marocain recense ainsi près de 130 opérateurs mais il est en fait dominé par Menara, marque commerciale de l'Internet de l'opérateur historique, avec une part de marché de $88 \%$ tous accès confondus (et $94 \%$ de l'ADSL). La concentration de ce marché des FAl est donc particulièrement préjudiciable aux opérateurs privés. Ceux-ci subissent des retards et des coûts d'interconnexion au backbone ${ }^{10}$ national, particulièrement élevés, imposés par l'opérateur historique qui contrôle cette infrastructure. Des coûts qui vont ensuite être répercutés par les FAI sur les tarifs d'abonnement qui deviendront trop onéreux pour séduire de nouveaux abonnés. Dans ces conditions, le risque à moyen terme est que le faible nombre d'abonnés se traduise par une rentabilité insuffisante pour les opérateurs privés. Ces derniers pourraient alors cesser leur activité et, par ricochet, entraîner une concentration plus forte du marché.

Par ailleurs, l'ouverture difficile du marché de la téléphonie fixe compromet également le développement de l'Internet. Au Maroc comme en Algérie, les premiers appels d'offres lancés en 2001-2002 ne suscitent que peu d'intérêt de la part des investisseurs privés locaux et étrangers. Ces derniers sont découragés par les conditions d'attribution des licences particulièrement dissuasives et des perspectives incertaines. D'autres appels d'offres, qui assouplissent les conditions d'attribution, sont donc lancés en 2004- 2005. Les licences se font aussi beaucoup moins onéreuses, ce qui leur permet de trouver preneurs pour un coût nettement inférieur (en millions d'euros, 50 en Algérie, 6,7 au Maroc) à celui des licences mobiles (Maroc : 1 milliard d'euros et 500 millions pour l'Algérie).

\subsection{Une logique de diffusion spécifique?}

Des logiques spécifiques, liées à la culture, président-elles à la diffusion africaine ? En quoi le déterminisme technologique répond-il à une conception

\footnotetext{
${ }^{10}$ Les backbones forment la colonne vertébrale d'Internet. Reliant les villes et pays entre eux, ils permettent de faire transiter l'information (mail, web, etc.) d'un endroit à un autre. En effet, un ordinateur est relié à son FAI, lui-même relié au reste du monde par le biais de backbones qui lui permettent de faire transiter la masse d'informations montantes et descendantes de ses clients.
} 
industrielle et peut-il, au bout du compte, influencer les politiques de diffusion ? Qu'en est-il des dynamiques sociales ?

\subsubsection{Le déterminisme comme logique de diffusion}

La diffusion des technologies en Afrique repose non seulement sur le postulat du déterminisme technologique, mais aussi sur l'idée selon laquelle il convient de pallier le sous-équipement par le biais d'une politique infrastructurelle globale. Faut-il souligner que l'OCDE considère que «les nouvelles technologies rendent l'administration publique plus efficace et l'Éducation de meilleure qualité ${ }^{11}$ ? Dans le même ordre d'idée, les déclarations au SMSI de Genève et de Tunis ne prêtent pas davantage à confusion. Est-il utile de rappeler que la devise du sommet de Genève était les "TIC pour le développement», tandis que le mot d'ordre de Tunis était "les TIC pour tous »? Ainsi comprend-on aisément que les stratégies de diffusion sont inséparables des stratégies d'équipement.

Les politiques d'aide ne dérogent pas à cette règle. Elles sont elles-mêmes souvent pétries de déterminisme. II n'est que de voir l'initiative "One laptop per child ", médiatisée à satiété et présentée comme susceptible de faciliter l'accès à la technologie. Or, s'agissant de ce projet, plusieurs aspects laissent perplexes. C'est d'abord l'idée de la possession d'un ordinateur personnel, fût-il à bas coût, qui pose question dans un contexte où l'utilisation du microordinateur est rarement individuelle mais principalement partagée. C'est ensuite l'idée du financement de ces machines par l'État qui semble assez peu réaliste, certains pays rencontrant déjà de grandes difficultés pour scolariser les enfants. Des doutes se font également jour en ce qui concerne les projets d'équipements d'envergure. Que cela concerne le plan «e-Schools » du Nepad dont l'objectif est d'équiper l'ensemble des écoles primaires et des établissements secondaires d'Afrique en ordinateurs d'ici 2025, ou les programmes de déploiement massif de machines low cost, comme les classMate de l'industriel Intel, beaucoup s'interrogent sur le financement et la pérennité de telles actions.

Le constat est similaire en ce qui concerne l'équipement. Si les ordinateurs de seconde main, très répandus en particulier en Afrique subsaharienne, servent fréquemment à équiper les cybercentres privés qui émergent en quantité dans les agglomérations, le recours aux machines d'occasion devient " très discutable avec la récente disponibilité d'ordinateurs neufs bon marché " [BAD / OCDE, 2009]. Quant aux logiciels libres, il semble qu'ils soient en butte à

${ }^{11}$ OCDE, Perspectives économiques en Afrique, 2009. 
Le développement des télécommunications dans les Suds

Retour sur une décennie de diffusion des TIC en Afrique de l'Ouest et au Maghreb

la méfiance des utilisateurs car moins performants que les logiciels standards piratés à partir de copies importées ${ }^{12}$.

Quoi qu'il en soit, les individus ne s'approprient pas les technologies parce qu'elles sont disponibles sur le marché, mais parce qu'elles apportent des réponses à des besoins personnels.

\subsubsection{Un consommateur atypique?}

La littérature sur la question, qu'elle soit d'essence universitaire ou journalistique, regorge d'expériences spécifiques et souvent inédites. En créant des précédents intéressants, de nombreux usages ont force d'exemple. D'aucuns proposent d'ailleurs de se fonder sur ces logiques spécifiques pour diffuser les technologies. Ceux dont se repaissent le plus la littérature spécialisée et les organismes internationaux ont trait aux dynamiques sectorielles, notamment dans le secteur de l'agriculture et de la pêche. C'est le cas d'une application mise au point par la société Manobi qui permet à des agriculteurs de recevoir en temps réel sur leur mobile les prix du kilo de fruits ou de légumes tels qu'ils sont pratiqués sur les marchés des grandes villes. Ainsi informés sur les prix, ces agriculteurs peuvent mieux négocier face aux grossistes qui viennent acheter leur production. Le modèle économique serait viable puisque les gains réalisés permettraient aux producteurs de s'abonner pour un coût variant entre 10 et 40 euros par mois. Autre originalité de l'application: la lecture se fait par un système d'icônes permettant aux analphabètes d'en tirer profit. D'après ses promoteurs ${ }^{13}$, ce programme devait toucher 600000 agriculteurs en 2011. Si ces chiffres, difficilement vérifiables, sont à considérer avec prudence, il n'en demeure pas moins qu'après le Sénégal, premier pays à avoir testé l'application, quelques États dont l'Afrique du Sud ont souhaité l'expérimenter.

Globalement, le spectre d'utilisation du téléphone mobile s'étend. II touche de plus en plus les sphères du quotidien. Les opérateurs privés ont en effet su s'adapter aux contraintes économiques de la région: le système des cartes prépayées convient à un continent où le taux de bancarisation est encore relativement faible. Les procédés permettant le partage des crédits téléphoniques entre abonnés existent dans plusieurs pays tels que le Mali ou

12 Selon International Data Company, le taux de piratage en Afrique est supérieur à $80 \%$ en 2004 , voir http://www.bsa.org

${ }^{13}$ Marchés tropicaux, n3112, 15 juillet 2005. 
Ahmed DAHMANI et Jean-Michel LEDJOU

l'Algérie. La possibilité d'acheter quelques unités téléphoniques dans des échoppes ou à des hommes sandwichs qui déambulent dans les rues témoigne de l'insertion des opérateurs dans le tissu socio-économique local. Par ailleurs, en dépit de la faiblesse des revenus, on se heurte parfois à des paradoxes. Dans un contexte où les subventions de terminaux ne sont que peu répandues, nombre de jeunes gens s'arrachent à prix fort des téléphones mobiles disposant des fonctionnalités les plus récentes. Des portables de contrefaçon s'écoulent tandis que d'autres, importés de Dubaï ou de Chine, trouvent des acquéreurs à des tarifs représentant plusieurs dizaines d'euros. Le rôle de la diaspora n'est pas neutre non plus : en envoyant des téléphones portables sur le continent, elle facilite l'acquisition de modèles de marque à bon compte. Enfin, pour l'instant cantonné à quelques pays, l'Internet mobile se développe sur des téléphones à des prix de plus en plus accessibles. L'extension de la technologie $3 G$ devrait permettre de démultiplier cette forme d'accès au Web.

Les logiques culturelles qui éclairent la diffusion commencent donc à s'éclaircir: au bricolage des usages succèdent des pratiques démultipliées. L'horizon du portable ayant fonction de " couteau suisse » s'est élargi au monde entier. Les technologies convergent et les usages se mutualisent. L'exemple du site Internet Ushahidi le prouve ${ }^{14}$. Initiative africaine dont le but est de faire connaître et de cartographier les actes de violence, l'application centralise des informations envoyées par des utilisateurs depuis leur téléphone mobile. «Des versions similaires de ce programme conçu à partir de logiciels libres ont servi en Inde à suivre les élections, en Afrique à signaler les pénuries de médicaments, au Moyen-Orient à recenser les actes de violence commis en temps de guerre et, à Washington, à signaler les routes bloquées et les endroits où trouver chasse-neige et souffleuses". On voit donc bien comment- des usages au départ spécifiques s'hybrident, se jouent des frontières et tendent à se mondialiser.

II n'en reste pas moins que les initiatives en termes de libéralisation et de privatisation du secteur des télécommunications, inspirées par différents organismes et instances internationaux au cours de ces dix dernières années, devaient permettre le développement et la modernisation des infrastructures, et accroître la connectivité des pays d'Afrique de l'Ouest et du Maghreb aux réseaux. L'indifférenciation des politiques de diffusion dans ce large espace, s'appuyant sur une sorte de mise en récit de la singularité des usages storytelling qui mériterait d'ailleurs d'être étudiée -, a conduit à une sorte de prosélytisme technologique. Dans les faits, et dans la majorité des cas,

14 Voir l'article de A. Giridharadas, "Africa's gift to Sillicon Valley : how to track a crisis », New York Times du 12 mars 2010,

tic\&société - 5 (2-3), $2^{\text {ème }}$ semestre $2011-1^{\text {er }}$ semestre 2012 
Le développement des télécommunications dans les Suds

Retour sur une décennie de diffusion des TIC en Afrique de l'Ouest et au Maghreb

l'ouverture des marchés a essentiellement profité aux opérateurs internationaux qui ne se sont intéressés qu'aux segments lucratifs et aux clients urbains. C'est ce qui pourrait expliquer l'essor prodigieux de la téléphonie cellulaire en Afrique de l'Ouest et au Maghreb, même si ce système rend par ailleurs des services considérables aux agents là où les réseaux filaires sont inexistants ou obsolètes. Le développement des télécommunications en Afrique rouvre le débat sur le rôle stratégique de l'État car la gestion du secteur des TIC ne peut être dominée par la seule sphère privée et par la seule logique marchande.

\section{Références bibliographiques}

Aïta. S., 2003-2004. «Internet en langue arabe : espace de liberté ou fracture sociale ? " Maghreb Machrek, n 978

Bahi A., 2004. « Usages d'Internet et logiques d'adaptation sociale des jeunes. Une étude dans les "cybercafés abidjanais" ", Bulletin du CODESRIA, $\mathrm{n} 9182$

Chéneau-Loquay A. (s/d), 2009. "Accès aux nouvelles technologies en Afrique et en Asie. TIC et service universel ", Netsuds, 4.

Chéneau-Loquay A. (s/d), 2004. Mondialisation et technologies de la communication en Afrique, Paris : Karthala.

Flichy P. 2005. "Le dévoppement du téléphone, leçons de l'histoire », in : Benamrane $\mathrm{D}$. et al. (s/d), Les télécommunications entre bien public et marchandise. Paris : Éditions Charles Léopold Mayer.

Kiyindou A. (s/d), 2009. Fractures, mutations, fragmentations, de la diversité des cultures numériques. Paris : Hermès ; Lavoisier.

Gabas J-J. (s/d), 2004. Société numérique et développement en Afrique. Usages et politiques publiques, Paris : Karthala.

Gonzalès-Quijano Y., 2002. "La révolution de l'information arabe aura-t-elle lieu ? » Politique étrangère, $\mathrm{n}$ 9, IFRI.

Hibou B., Tozy M., janvier 2002. « De la friture sur la ligne des réformes », Critique Internationale, $\mathrm{n} 94$

Mermier F. (ed.), 2003. Mondialisation et nouveaux médias dans l'espace arabe, Paris : Maisonneuve-Larose 
Mezouaghi M., (s/d), 2007. Le Maghreb dans l'économie numérique, IRMC, Paris : Maisonneuve-Larose.

Mihoub-Dramé.S., 2005. Internet dans le monde arabe, Paris : L'Harmattan.

Ntambue Tshimbulu R., 2001. L'Internet, son web et son e-mail en Afrique. Approche critique, Paris : L'Harmattan.

Sagna O. 2007. "Société civile et construction de la Société de l'information en Afrique de l'Ouest : entre marginalisation et implication » in : Dahmani A. et al (s/d), La démocratie à l'épreuve de la société numérique, Paris : Karthala

Tcheng. H., Huet. J.-M., Viennois. I., Romdhane. M., février 2009.

"Télécommunications et développement en Afrique », Futuribles, n 349.

Tcheng H., Huet J.-M., Romdhane M., février 2010, Les enjeux financiers de l'explosion des télécoms en Afrique subsaharienne, IFRI.

\section{Rapports}

BAD/OCDE, Perspectives économiques en Afrique, 2009

CNUCED, Rapport 2007-2008 sur I économie de l'information.- Science et technologie pour le développement : le nouveau paradigme des TIC, Nations Unies, 2007.

Mezouaghi. M, Libéralisation des services de télécommunication au Maghreb: transition institutionnelle et performances, AFD, décembre 2005.

UIT, Indicateurs des télécommunications/TIC Africaines : à la croisée des chemins, 2008.

UIT, Telecommunication/ICT, Markets and trends in Africa, 2007

World Association of Newspapers, World Press Trends, Paris-Montréal, 2005 